\title{
FUZZY VERSION OF SECANT METHOD TO SOLVE FUZZY NON-LINEAR EQUATIONS
}

\author{
Goutam Kumar Saha ${ }^{1}$ and Shapla Shirin ${ }^{2}$ \\ ${ }^{1}$ Department of Mathematics, Jagannath University \\ ${ }^{2}$ Department of Mathematics, University of Dhaka \\ Corresponding author: shapla@du.ac.bd
}

Received 24.09.2014 Accepted 10.11.2015

\begin{abstract}
In this paper fuzzy version of secant method has been introduced to obtain approximate solutions of a fuzzy non-linear equation. Graphical representations of the approximate solutions have also been shown. The idea of converging to the root to the desired degree of accuracy, which is the optimum solution, of a fuzzy non-linear equation has been focused.
\end{abstract}

Keywords: Fuzzy version of Secant method, Fuzzy non-linear equation (FNLE), Linear fuzzy real number $(L F R N)$

\section{Introduction}

Non-linear equations have played an important role to develop mathematical modeling in different discipline, such as, in engineering, mathematics etc. Numerical approximation methods are usually needed in solving the non-linear equations of the form $f(x)=0$, because it is not always possible to get the exact solutions of equations. On the other hand, the parameters of a non-linear equation are not always represented by crisp numbers, but also by fuzzy numbers. A non-linear equation over linear fuzzy real numbers is called a fuzzy non-linear equation, that is, an equation of the form $f\left(\mu_{x}\right)=0$ is called a fuzzy non-linear equation of fuzzy variable. Triangular fuzzy numbers and trapezoidal fuzzy numbers are called the linear fuzzy real numbers. In this paper triangular fuzzy numbers are used as linear fuzzy real numbers.

Different methods have been used in solving fuzzy non-linear equations [6, 8, 9]. In [3] secant method has been used to solve the same in which the coefficients are fuzzy numbers, but the variables are not fuzzy numbers. In this paper fuzzy version of secant method has been introduced to solve a fuzzy non-linear equation where the coefficients and the variables are fuzzy numbers. Therefore, the limitation of [3] has been overcome by introducing this new method. Three examples have also been discussed and the approximate solutions, which are LFRNs, computed from each iteration, are shown in a tabular form. The graphical representations of approximate solutions are depicted to achieve the idea of converging to the root, an optimum solution, of the FNLE. 
Some related definitions and some operations on linear fuzzy real numbers [2, 5-9], which will be used later on to obtain a solution of fuzzy nonlinear equation to any desired degree of accuracy, has been given in the following section.

\section{Preliminaries}

In this section some definitions have been discussed that are important for representing our main objective in the later sections.

Definition 2.1 (Linear fuzzy real number, $L F R N$ ) [6] Let $\mathfrak{R}$ be the set of all real numbers. Then a linear fuzzy real number $\mu$ with associated triple of real numbers $(a, b, c)$ is a triangular fuzzy number, denoted by $\mu(a, b, c)$, whose membership function $\mu: \Re \rightarrow[0,1]$ is defined by

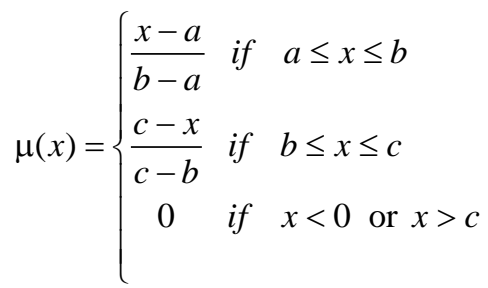

where $a \leq b \leq c$, shown in Fig.1.1.

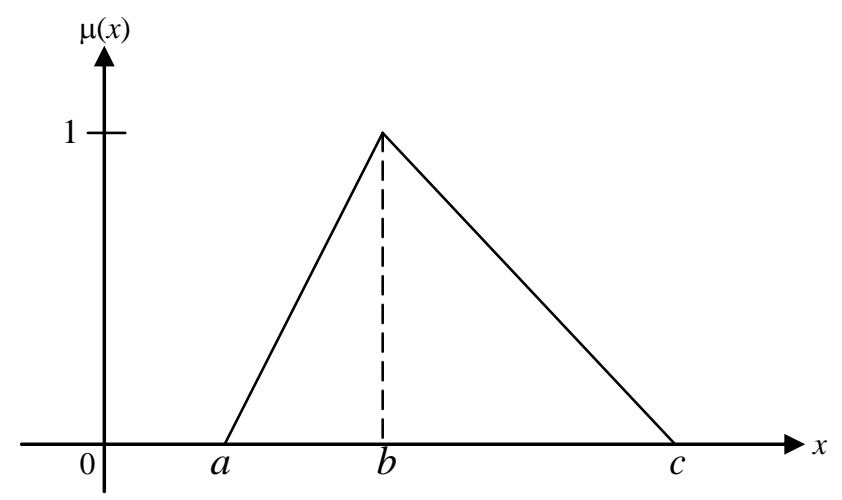

Fig. 1.1 Linear fuzzy real number $\mu(a, b, c)$.

Let $L F^{\Re}$ be the set of all linear fuzzy real numbers. Note that any real number $b$ can be written as a linear fuzzy real number, $r(b)$, where $r(b)=\mu(b, b, b)$ and therefore $\mathfrak{R} \subseteq L F^{\Re}$. It is clear that $r(b)$ represents the real number $b$ itself. Operations on $L F R N$, fuzzy functions and fuzzy linear equations in $L F^{\Re}$ are also defined in $L F^{\Re}$ as follows:

Definition 2.2. (Operations on $L F^{\Re}$ ) [6] For given two linear fuzzy real numbers $\mu_{1}=\mu\left(a_{1}, b_{1}, c_{1}\right)$ and $\mu_{2}=\mu\left(a_{2}, b_{2}, c_{2}\right)$, we define addition, subtraction, multiplication and division by

1. $\mu_{1}+\mu_{2}=\mu\left(a_{1}+a_{2}, b_{1}+b_{2}, c_{1}+c_{2}\right)$;

2. $\quad \mu_{1}-\mu_{2}=\mu\left(a_{1}-c_{2}, b_{1}-b_{2}, c_{1}-a_{2}\right)$; 
3. $\mu_{1} \cdot \mu_{2}=\mu\left(\min \left\{a_{1} a_{2}, a_{1} c_{2}, a_{2} c_{1}, c_{1} c_{2}\right\}, b_{1} b_{2}, \max \left\{a_{1} a_{2}, a_{1} c_{2}, a_{2} c_{1}, c_{1} c_{2}\right\}\right)$

4. $\frac{\mu_{1}}{\mu_{2}}=\mu_{1} \cdot \frac{1}{\mu_{2}}$, where $\frac{1}{\mu_{2}}=\left(\min \left\{\frac{1}{a_{2}}, \frac{1}{b_{2}}, \frac{1}{c_{2}}\right\}\right.$, median $\left.\left\{\frac{1}{a_{2}}, \frac{1}{b_{2}}, \frac{1}{c_{2}}\right\}, \max \left\{\frac{1}{a_{2}}, \frac{1}{b_{2}}, \frac{1}{c_{2}}\right\}\right)$ and $a_{2} \neq 0, b_{2} \neq 0, c_{2} \neq 0$.

Definition 2.3. (Function in $L F^{\Re}$ ) [6] If $f: \mathfrak{R} \rightarrow \mathfrak{R}$ is a real-valued function and $\mu(a, b, c)$ is a $L F R N$, then the linear fuzzy real-valued function $\bar{f}: L F^{\Re} \rightarrow L F^{\Re}$ is defined as

$$
\bar{f}(\mu(a, b, c))=\mu(\bar{a}, \bar{b}, \bar{c})
$$

where $\bar{a}=\min \{f(a), f(b), f(c)\}, \bar{b}=\operatorname{median}\{f(a), f(b), f(c)\}$, and

$\bar{c}=\max \{f(a), f(b), f(c)\}$. We note that if $a=b=c$ then $\bar{a}=\bar{b}=\bar{c}$, i.e., $\bar{f}(r(b))=r(f(b))$. Here $\bar{f}$ is an extension of the function $f$.

Definition 2.4. (Linear equation in $L F^{\Re}$ ) [6] A linear equation over $L F^{\Re}$ is an equation of the form

$$
\mu_{1} \cdot \mu_{x}+\mu_{2}=\mu_{3}
$$

where the $\mu_{i}$ are $L F R N$ s for $i=1,2,3$ with a triple of unknown real numbers $\left(\alpha_{i}, \beta_{i}, \gamma_{i}\right)$.

Definition 2.5. (Fuzzy non-linear equations in $L F^{\Re}$ ) [6] $\bar{f}\left(\mu_{x}\right)=0$ is called a fuzzy non-linear equation where $\bar{f}: L F^{\Re} \rightarrow L F^{\Re}$ is a fuzzy non-linear function, i.e., . $\bar{f}: L F^{\Re} \rightarrow L F^{\Re}$ is non-linear function of fuzzy variable. Here $\mu_{x}$ in $L F^{\Re}$ is a solution of a fuzzy a non-linear equation $\bar{f}\left(\mu_{x}\right)=0$.

For example $\mu_{x}^{3}-2 \mu_{x}-5=0$ is fuzzy non-linear equation.

\section{The Fuzzy Version of Secant Method to Solve Fuzzy Non-Linear Equation}

In this section, we are going to discuss how to solve fuzzy non-linear equations in $L F^{\Re}$. Solving fuzzy non-linear equations over $L F^{\Re}$ of the form $\bar{f}\left(\mu_{x}\right)=0$ is possible with a modification of Newton's method over linear fuzzy real numbers. The classical form of Newton's method has been discussed in $[1,10]$. The formula of fuzzified form of Newton's method is given below [6]:

$$
\mu_{x}^{(n+1)}=\mu_{x}^{(n)}-\frac{\bar{f}\left(r\left(b^{(n)}\right)\right)}{\overline{f^{\prime}}\left(r\left(b^{(n)}\right)\right)}, \text { for } n \geq 0
$$

This method is required an initial approximation in $L F^{\Re}$, say, $\mu_{x}^{(0)}=\mu\left(a^{(0)}, b^{(0)}, c^{(0)}\right)$. 
From formula (1) it is clear that Newton's method requires the evaluation of derivatives of the function. Sometimes it is not possible of computing the derivative, particularly in the case of functions arising in practical problems. Classical form of computing the derivative of a function at any point is used in the Secant method [10]. The fuzzified form of the derivative at $\mu_{x}^{(n)}$ is approximated by the formula

$$
\overline{f^{\prime}}\left(r\left(b^{(n)}\right)\right)=\frac{\bar{f}\left(r\left(b^{(n)}\right)\right)-\bar{f}\left(r\left(b^{(n-1)}\right)\right)}{\mu_{x}^{(n)}-\mu_{x}^{(n-1)}}
$$

Thus, the equation (1) reduces to

$$
\mu_{x}^{(n+1)}=\mu_{x}^{(n)}-\frac{\bar{f}\left(r\left(b^{(n)}\right)\right)\left(\mu_{x}^{(n)}-\mu_{x}^{(n-1)}\right)}{\bar{f}\left(r\left(b^{(n)}\right)\right)-\bar{f}\left(r\left(b^{(n-1)}\right)\right)}, \text { for } n \geq 0
$$

The new formula, shown in (3), is the fuzzy version of Secant formula to solve the fuzzy nonlinear equation $\bar{f}\left(\mu_{x}\right)=0$ and this method is the fuzzy version of Secant method. This method begins with two initial approximations $\mu_{x}^{(-1)}=\mu\left(a^{(-1)}, b^{(-1)}, c^{(-1)}\right)$ and $\mu_{x}^{(0)}=\mu\left(a^{(0)}, b^{(0)}, c^{(0)}\right) \in L F^{\Re}$. Then successive approximations of the solution using fuzzy version of Secant method are

$$
\mu_{x}^{(1)}=\mu_{x}^{(0)}-\frac{\bar{f}\left(r\left(b^{(0)}\right)\right)\left(\mu_{x}^{(0)}-\mu_{x}^{(-1)}\right)}{\bar{f}\left(r\left(b^{(0)}\right)\right)-\bar{f}\left(r\left(b^{(-1)}\right)\right)}, \mu_{x}^{(2)}=\mu_{x}^{(1)}-\frac{\bar{f}\left(r\left(b^{(1)}\right)\right)\left(\mu_{x}^{(1)}-\mu_{x}^{(0)}\right)}{\bar{f}\left(r\left(b^{(1)}\right)\right)-\bar{f}\left(r\left(b^{(0)}\right)\right)}, \text { and so on, }
$$

where $r\left(b^{n}\right)=\mu\left(b^{n}, b^{n}, b^{n}\right)$ and $\bar{f}\left(r\left(b^{(n)}\right)\right)$ is the value of $\bar{f}$ at $r\left(b^{n}\right)=\mu\left(b^{n}, b^{n}, b^{n}\right)$. The stopping criterion of this method is $\left|r\left(b^{(n)}-b^{(n-1)}\right)\right|<\varepsilon$, where $\varepsilon$ is preset small value, for example $\varepsilon=0.0000001$. A solution of a fuzzy non-linear equation $\bar{f}\left(\mu_{x}\right)=0$ exists if and only if the sequence of approximate solutions $\left\{\mu_{x}^{(n)}\right\}_{n=1}^{\infty}$ converses to a fuzzy number if the stopping criterion is satisfied and the limit of the sequence is an optimal solution of the fuzzy non-linear equation $\bar{f}\left(\mu_{x}\right)=0$.

\section{Algorithm of Fuzzy Version of Secant Method}

Two initial approximations $\mu_{x}^{(-1)}=\mu\left(a^{(-1)}, b^{(-1)}, c^{(-1)}\right)$ and $\mu_{x}^{(0)}=\mu\left(a^{(0)}, b^{(0)}, c^{(0)}\right)$ are needed to find a solution to $\bar{f}\left(\mu_{x}\right)=0$. The algorithm of fuzzy version of Secant method is as under:

INPUT: Initial approximations $\mu_{x}^{(-1)}=\mu\left(a^{(-1)}, b^{(-1)}, c^{(-1)}\right), \mu_{x}^{(0)}=\mu\left(a^{(0)}, b^{(0)}, c^{(0)}\right)$; tolerance TOL; maximum number of iterations $n$.

OUTPUT: approximation solution $\mu_{x}^{(n)}=\mu\left(a^{(n)}, b^{(n)}, c^{(n)}\right)$ or message of failure. 
Step-1: Set $i=0$.

Step-2: While $i \leq n$ do steps 3 - 6 .

Step-3: Set $\mu_{x}^{(i+1)}=\mu_{x}^{(i)}-\frac{\bar{f}\left(r\left(b^{(i)}\right)\right)\left(\mu_{x}^{(i)}-\mu_{x}^{(i-1)}\right)}{\bar{f}\left(r\left(b^{(i)}\right)\right)-\bar{f}\left(r\left(b^{(i-1)}\right)\right)} \cdot \quad$ (Compute $\left.\mu_{x}^{(i+1)}\right)$

Step-4: If $\left|r\left(b^{(i)}-b^{(i-1)}\right)\right|<T O L$ then

OUTPUT $\mu_{x}^{(i+1)}$.

(The procedure was successful.)

STOP.

Step-5: Set $i=i+1$

Step-6: $\mu_{x}^{(i-1)}=\mu_{x}^{(i)}$

(Update $\mu_{x}^{(-1)}$ )

Step-7: OUTPUT. ("The method failed after $n$ iteration.");

(The procedure is unsuccessful after $n$ iteration.)

STOP.

Example 1. Solve the fuzzy non-linear equation $\mu_{x}^{3}-2 \mu_{x}-5=0$.

Solution: Let $\bar{f}\left(\mu_{x}\right)=\mu_{x}^{3}-2 \mu_{x}-5$. The fuzzy version of Secant method is given by the formula

$$
\mu_{x}^{(n+1)}=\mu_{x}^{(n)}-\frac{\bar{f}\left(r\left(b^{(n)}\right)\right)\left(\mu_{x}^{(n)}-\mu_{x}^{(n-1)}\right)}{\bar{f}\left(r\left(b^{(n)}\right)\right)-\bar{f}\left(r\left(b^{(n-1)}\right)\right)}, \text { for } n \geq 0
$$

Using initial approximations $\mu_{x}^{(-1)}=\mu(1,2,3)$ and $\mu_{x}^{(0)}=\mu(2,3,4)$, we obtain the approximate solutions of the fuzzy non-linear equation which are shown in the following table:

Table. 1. Approximate solutions of fuzzy non-linear equation.

\begin{tabular}{|c|c|c|l|}
\hline$n$ & \multicolumn{2}{|c|}{ Initial approximations } & \multicolumn{1}{|c|}{ Approximations $\mu_{x}^{(n)}$} \\
\hline 1. & $\mu_{x}^{(-1)}$ & $\mu_{x}^{(0)}$ & $\mu(-0.823529412,2.05882353,4.94117647)$ \\
\hline 2. & $\mu_{x}^{(0)}$ & $\mu_{x}^{(1)}$ & $\mu(-0.893654817,2.081263659,5.056182135)$ \\
\hline 3. & $\mu_{x}^{(1)}$ & $\mu_{x}^{(2)}$ & $\mu(-4.419621793,2.094824145,8.609270086)$ \\
\hline 4. & $\mu_{x}^{(2)}$ & $\mu_{x}^{(3)}$ & $\mu(-4612135961,2.094549432,8.801234826)$ \\
\hline 5. & $\mu_{x}^{(3)}$ & $\mu_{x}^{(4)}$ & $\mu(-4.710753763,2.094551481,8.899856731)$ \\
\hline
\end{tabular}

Thus at iteration $5, \mu_{x}^{(5)}=\mu(-4.710753768,2.094551481,8.899856731)$ is the final approximation as optimum solution which is correct up to 8 decimal places. The graphical representations of the approximate solutions and the optimum solution with desired accuracy are shown in the following figures. 


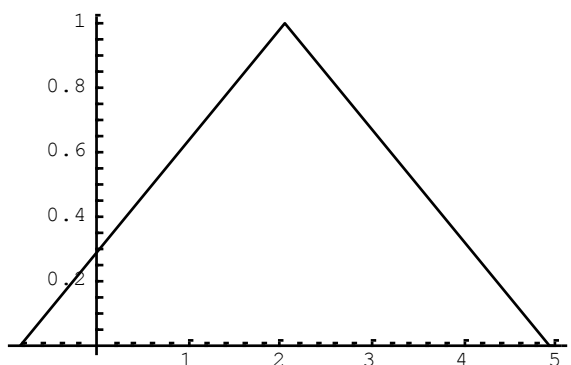

Iteration-1

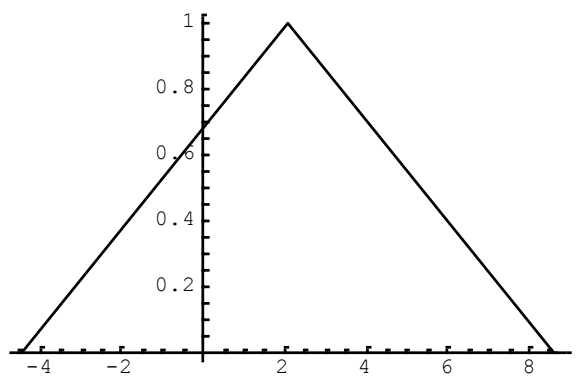

Iteration-3

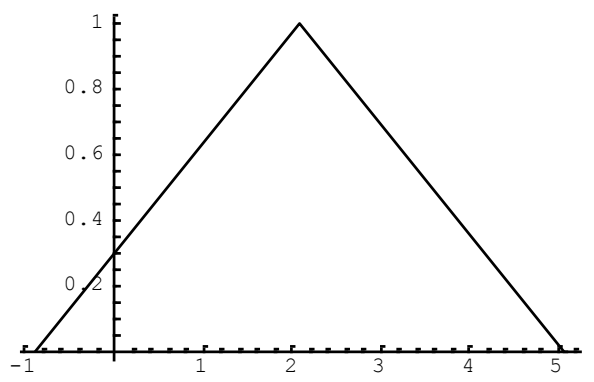

Iteration-2

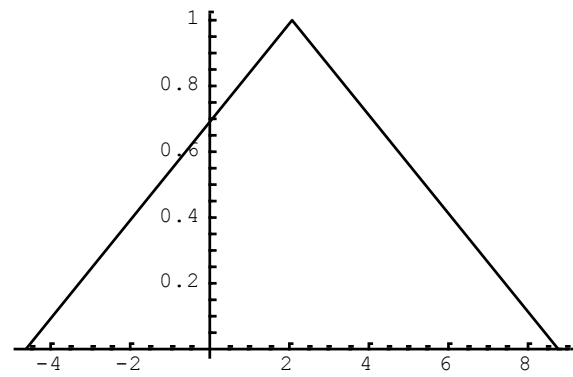

Iteration-4

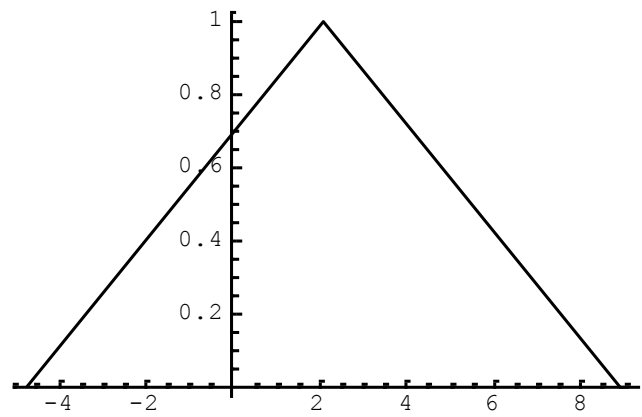

Iteration-5

Fig 1.2: Graphical representations of approximate solutions of the given fuzzy non-linear equation.

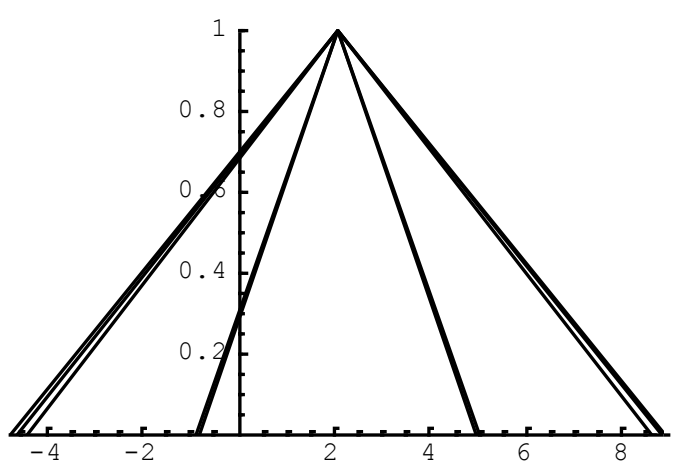

Fig.1.3. Graphical representation of optimum solution of fuzzy non-linear equation of Example 1. 
Example 2. Solve the fuzzy non-linear equation $\mu_{x}^{3}+\mu_{x}^{2}-1=0$.

Solution: Let $\bar{f}\left(\mu_{x}\right)=\mu_{x}^{3}+\mu_{x}^{2}-1$. From the fuzzy version of Secant method we have

$$
\mu_{x}^{(n+1)}=\mu_{x}^{(n)}-\frac{\bar{f}\left(r\left(b^{(n)}\right)\right)\left(\mu_{x}^{(n)}-\mu_{x}^{(n-1)}\right)}{\bar{f}\left(r\left(b^{(n)}\right)\right)-\bar{f}\left(r\left(b^{(n-1)}\right)\right)}, \text { for } n \geq 0
$$

Using initial approximations $\mu_{x}^{(-1)}=\mu(0,0.5,1) \quad$ and $\quad \mu_{x}^{(0)}=\mu(0.5,1,1.5)$, the obtained approximations of the solution are shown in Table 2:

Table. 2. Approximate solutions of fuzzy non-linear equation.

\begin{tabular}{|c|c|c|l|}
\hline$n$ & \multicolumn{2}{|c|}{ Initial approximations } & \multicolumn{1}{|c|}{ Approximations $\mu_{x}^{(n)}$} \\
\hline 1. & $\mu_{x}^{(-1)}$ & $\mu_{x}^{(0)}$ & $\mu(-0.423076923,0.692307693,1.807692307)$ \\
\hline 2. & $\mu_{x}^{(0)}$ & $\mu_{x}^{(1)}$ & $\mu(-0.630845798,0.741194487,2.11323477)$ \\
\hline 3. & $\mu_{x}^{(1)}$ & $\mu_{x}^{(2)}$ & $\mu(-1.359145647,0.755795138,2.870735922)$ \\
\hline 4. & $\mu_{x}^{(2)}$ & $\mu_{x}^{(3)}$ & $\mu(-1.58227957,0.75486482,3.091987595)$ \\
\hline 5. & $\mu_{x}^{(3)}$ & $\mu_{x}^{(4)}$ & $\mu(-1.643663943,0.754877654,3.15341925)$ \\
\hline
\end{tabular}

Thus at iteration $5, \mu_{x}^{(5)}=\mu(-1.643663943,0.754877654,3.15341925)$ is the final approximation which is correct up to 7 decimal places.

Example 3. Solve the fuzzy non-linear equation $\mu_{x} e^{\mu_{x}}-1=0$.

Solution: Let $\bar{f}\left(\mu_{x}\right)=\mu_{x} e^{\mu_{x}}-1$. From the fuzzy version of Secant method we have

$$
\mu_{x}^{(n+1)}=\mu_{x}^{(n)}-\frac{\bar{f}\left(r\left(b^{(n)}\right)\right)\left(\mu_{x}^{(n)}-\mu_{x}^{(n-1)}\right)}{\bar{f}\left(r\left(b^{(n)}\right)\right)-\bar{f}\left(r\left(b^{(n-1)}\right)\right)}, \text { for } n \geq 0
$$

Using initial approximations $\mu_{x}^{(-1)}=\mu(0.25,0.5,0.75)$ and $\mu_{x}^{(0)}=\mu(0.5,0.75,1)$, the obtained approximations of the solution are shown in Table 3:

Table. 3. Approximate solutions of fuzzy non-linear equation.

\begin{tabular}{|c|c|c|l|}
\hline$n$ & \multicolumn{2}{|c|}{ Initial approximations } & \multicolumn{1}{|c|}{ Approximations $\mu_{x}^{(n)}$} \\
\hline 1. & $\mu_{x}^{(-1)}$ & $\mu_{x}^{(0)}$ & $\mu(-0.07744124,0.30751958,1.19248041)$ \\
\hline 2. & $\mu_{x}^{(0)}$ & $\mu_{x}^{(1)}$ & $\mu(-0.4219077,0.52762641,1.9485481)$ \\
\hline 3. & $\mu_{x}^{(1)}$ & $\mu_{x}^{(2)}$ & $\mu(-0.78045042,0.57651038,2.3985042)$ \\
\hline 4. & $\mu_{x}^{(2)}$ & $\mu_{x}^{(3)}$ & $\mu(-1.3385738,0.56683687,3.737078)$ \\
\hline 5. & $\mu_{x}^{(3)}$ & $\mu_{x}^{(4)}$ & $\mu(-1.4805778,0.56714095,3.8545493)$ \\
\hline 6. & $\mu_{x}^{(4)}$ & $\mu_{x}^{(5)}$ & $\mu(-1.5207466,0.5671432909,3.8945292)$ \\
\hline
\end{tabular}


Thus at iteration $6, \mu_{x}^{(6)}=\mu(-1.5207466,0.5671432909,3.8945292)$ is the final approximation which is correct up to 9 places of decimal.

\section{Conclusion}

In this paper a new approach named fuzzy version of Secant method has been introduced in order to solve a fuzzy non-linear equation $(F N L E)$. By using the new approach, three fuzzy non-linear equations (FNLE) have been solved and obtained solutions of the FNLEs to the desired degree of accuracy which are the optimum solutions of the given equations. We have also represented the approximate solutions of the FNLE graphically so that one can achieve the idea of convergence of approximate solutions to the root to the required accuracy.

\section{REFERENCES}

[1] Burden R. L. and Faires J. D., Numerical Analysis, 7th edition, 2002, P. 69 - 73.

[2] Dubois D., Prade H., System of linear fuzzy constraints, Fuzzy Sets and Systems, 13, 1982, P. 1 - 10.

[3] Khorasani M. S., Solving Fuzzy Nonlinear Equation with Secant Method, International Journal of Algebra, 5(6), 2011, P. 295-299.

[4] Klir G. J., Yuan B., Fuzzy Sets and Fuzzy logic - Theory and Applications, Prentice-Hall of India Private Limited, New Delhi, 1997, P.1-117.

[5] Neggers J., Kim H., On Linear Fuzzy Real Numbers, Manuscript for book under development, 2007.

[6] Rogers F. and Jun Y., Fuzzy Nonlinear Optimization for the Linear Fuzzy Real Number System, International Mathematical Forum, 4, 2009, no. 12, P. 589 - 596.

[7] Rogers F., Neggers J., Jun Y., Method for optimizing linear problems with fuzzy constrains, International Mathematical Forum 3, 2008, P. 1141 - 1155.

[8] Saha G. K., Shirin S., A New Approach to Solve Fuzzy Non-Linear Equations using Fixed Point Iteration Algorithm, Ganit, J. Bangladesh Math. Soc., 32, 2012, P. 15-21.

[9] Saha G. K., Shirin S., Solution of Fuzzy Non-linear Equation using Bisection Algorithm, Dhaka Univ. J. Sci. 61(1), 2013(January), P.53-58.

[10] Sastry S. S., Introductory Methods of Numerical Analysis, 5th edition, 2012, P. 49-51. 